\title{
Germanica
}

philosophique

\section{Heiterkeit im Schatten der Weltkriege und des Holocaust}

Heiterkeit im Schatten der Weltkriege und des Holocaust

L'allégresse dans l'ombre des Guerres mondiales et de l'holocauste

Serenity in the Shadow of two World Wars and the Holocaust

\section{Helmuth Kiesel}

\section{(2) OpenEdition}

\section{Journals}

Édition électronique

URL : http://journals.openedition.org/germanica/5399

DOI : 10.4000/germanica.5399

ISSN : 2107-0784

Éditeur

Université de Lille

\section{Édition imprimée}

Date de publication : 31 décembre 2018

Pagination : 17-36

ISBN : 978-2-913857-42-1

ISSN : 0984-2632

Référence électronique

Helmuth Kiesel, „Heiterkeit im Schatten der Weltkriege und des Holocaust“, Germanica [Online], 63 |

2018, Online erschienen am: 01 Januar 2021, abgerufen am 22 Januar 2021. URL: http://

journals.openedition.org/germanica/5399; DOl: https://doi.org/10.4000/germanica.5399

(c) Tous droits réservés 


\title{
Heiterkeit im Schatten der Weltkriege und des Holocaust
}

\author{
Helmuth KIESEL \\ Universität Heidelberg
}

\section{Friedrich Schillers Heiterkeitspostulat}

Bekanntlich war es Friedrich Schiller, der den Begriff der Heiterkeit - verstanden als poetisch vermittelte Freiheit des Gemüts gegenüber der oft niederdrückenden Beschaffenheit des Lebens und der Geschichte ${ }^{1}$ ins Zentrum des klassischen und zugleich modernen Kunstverständnisses rückte. Eine besonders prägnante Stelle findet sich in der 1791 publizierten Auseinandersetzung mit Gottfried August Bürgers Gedichten, einem Artikel, der weit über eine Rezension hinausgeht und, indem er einen emphatischen Begriff von Dichtertum und Poesie entwickelt, Heiterkeit zur conditio sine qua non ideal(istisch)en Dichtertums und zugleich zum Wesenskern ideal(isierend)er Poesie erhebt. Gegen Ende dieses Artikels heißt es:

Nur die heitre, die ruhige Seele gebiert das Vollkommene. Kampf mit äußern Lagen und Hypochondrie, welche überhaupt jede Geisteskraft

1. - Vgl. Schillers Brief an Goethe vom 26. Dezember 1797 (Schillers Werke. Nationalausgabe, Weimar, Böhlau, 1977, Bd. 29, S. 177) und den zweiten der Briefe Über die ästhetische Erziehung, in: Friedrich Schiller, Sämtliche Werke. Hrsg. von Gerhard Fricke und Herbert G. Göpfert, München, Hanser, 1959, Hier Bd. 5: Erzählungen/ Theoretische Schriften, S. 572.

GERMANICA, 2018, LXIII, pp. 17 à 36. 
lähmen, dürfen am allerwenigsten das Gemüt des Dichters belasten, der sich von der Gegenwart loswickeln und frei und kühn in die Welt der Ideale emporschweben soll. Wenn es auch noch so sehr in seinem Busen stürmt, so müsse Sonnenklarheit seine Stirne umfließen².

Ein Jahr später hat Schiller sein Heiterkeitspostulat am Ende des Prologs zu seinem, ,finstern“ Geschichtsdrama Wallensteins Lager erneut formuliert und hat bei dieser Gelegenheit, indem er die Verwendung der Vers- und Reimform verteidigte, zugleich auf die Leistung der Form für das Zustandekommen der poetischen Heiterkeit hingewiesen. Die letzten Verse des Prologs lauten:

Und wenn die Muse heut,

Des Tanzes freie Göttin und Gesangs,

Ihr altes deutsches Recht, des Reimes Spiel,

Bescheiden wieder fordert - tadelts nicht!

Ja, danket ihrs, daß sie das düstre Bild

Der Wahrheit in das heitre Reich der Kunst

Hinüberspielt, die Täuschung, die sie schafft,

Aufrichtig selbst zerstört und ihren Schein

er Wahrheit nicht betrüglich unterschiebt;

Ernst ist das Leben, heiter ist die Kunst ${ }^{3}$.

Der sentenzhaft formulierte letzte Vers prägte das Kunstverständnis in Deutschland nachhaltig. Er wurde zum geflügelten Wort, das allenthalben zitiert wurde, wo es darum ging, das Verhältnis von Kunst und Leben zu bestimmen, und oft missbraucht wurde, um die ästhetischen und ethischen Ansprüche einer idealisierenden Kunst an das bürgerliche Leben abzuweisen und um Kunst als wohlfeiles Mittel der Ablenkung von den Problemen des ,wirklichen“ Lebens, als Medium der Zerstreuung und Entspannung oder als Feierabendvergnügen erscheinen zu lassen. Gleichviel, Kunst und Heiterkeit gehörten für die deutsche Autorenschaft - Philosophen eingeschlossen - wie für das deutsche Publikum eng zusammen; Harald Weinrich hat in seiner Kleinen Literaturgeschichte der Heiterkeit die wichtigsten Referenzen aufgeführt ${ }^{4}$, und Weiteres und Genaueres ist in philosophisch-ästhetischen Abhandlungen wie Walter Schulz' Metaphysik des Schwebens ${ }^{5}$ und in literaturgeschichtlichen Einzelstudien nachzulesen 6 . Benachbart ist dem Postulat der Heiterkeit

\footnotetext{
2. - Ebd., S. 985 .

3. - Ebd., Bd 2: Dramen, S. 274.

4. - Harald Weinrich, Kleine Literaturgeschichte der Heiterkeit, München, Beck,

5. - Walter Schulz, Metaphysik des Schwebens. Untersuchungen zur Geschichte der Ästhetik, Pfullingen, Neske, 1985.

6. - Genannt sei vor allem: Petra Kiedaisch/Jochen A. Bär (Hrsg.), Heiterkeit.
} 2001. 
das Postulat der Verklärung, wie es Gottfried Keller ${ }^{7}$ und Theodor Fontane $^{8}$ für die Zeit des Realismus geltend gemacht haben: Indem Mensch und Leben nicht ungeschminkt veristisch, sondern mit einer Tendenz zur adhortativ gemeinten Veredelung oder eben Verklärung dargestellt werden, bringt diese Heiterkeit ins Werk und lässt sie auf den Rezipienten überspringen.

\section{Schwierigkeiten mit dem Heiterkeitspostulat nach dem Ersten Weltkrieg}

Einen ersten Bruch mit dem Heiterkeitspostulat brachte der Naturalismus, der die „Brutalitäten“9 des Daseins „unerbittlich“, registrieren und zur Kenntnis bringen wollte; nicht umsonst erschrak Fontane bei aller Bewunderung für den jungen Gerhart Hauptmann über dessen erstes „,soziales Drama“ Vor Sonnenaufgang und schrieb am 8. September 1889 an seinen Verleger Paul Ackermann, die Literatur habe „,im Letzten“ doch „andre Aufgaben“, nämlich Verklärung und Erhebung von Mensch und Dasein. Erst recht in die Bredouille geriet das Heiterkeitspostulat aber mit dem Ersten Weltkrieg, dessen Zwangs- und Vernichtungsmaschinerie alle früheren Erfahrungen und Vorstellungen von Gewalt und Vernichtung in den Schatten stellten. Gewiss gab es Kriegsbücher, die auch noch über das Soldatenleben mit all seinen Strapazen und Schikanen, Entbehrungen und Destruktionserfahrungen auf humoristische Weise zu reden wussten; das Bumserbuch des Artilleristen Oskar Wöhrle, das von 1916 bis 1929 mehrfach aufgelegt wurde, ist ein literarisch durchaus bemerkenswertes Beispiel. Aber vielen Autoren war es angesichts des jahrelang sich hinziehenden Schlachtens und der schrecklichen Bilanz des Krieges - rund 10 Millionen Tote und 18 Millionen Versehrte - nicht möglich, ein „Gemüt“, wie Schiller sagte, zu bewahren, das es ihnen erlaubt hätte, Werke zu schaffen, die sowohl der Barbarei der jüngsten Geschichte als auch dem Heiterkeitspostulat gerecht geworden wären. Gottfried Benns geschichtsreflektorisches Gedicht Chaos von 1923 zeigt keine Spur von Heiterkeit und entbehrt jeden Hinweises auf einen positiven, befreienden oder versöhnlichen Gesichtspunkt. Thomas Mann, der während des Krieges bekanntlich mit der Feder tapfer für die deutsche Sache focht, nahm unmittelbar nach dem Krieg seine Zuflucht zu zwei Idyllen, in

Konzepte in Literatur und Geistesgeschichte, München, Fink, 1997.

7. - Brief vom 25. Juni 1860 an Berthold Auerbach. Vgl. https://www. gottfriedkeller.ch/briefe/frameset.php?https://www.gottfriedkeller.ch/briefe/auerbach. php.

8. - Brief vom 10. Oktober 1889 an Friedrich Spielhagen.

9. - So der Titel eines 1886 erschienenen Bandes mit „Skizzen und Studien“ von Hermann Conradi (Zürich, Schabelitz, 1886). 
denen der Krieg nur den atmosphärischen Hintergrund bildet, nämlich zu der Erzählung Herr und Hund sowie dem kleinen Versepos Gesang vom Kindchen ${ }^{10}$. Die Darstellung von Kriegshandlungen selbst mied Mann bis zu den letzten Absätzen seines 1924 abgeschlossenen und publizierten Romans Der Zauberberg, in denen sein Held Hans Castorp als Kriegsfreiwilliger wohl bei Langemarck ins Feuer gerät; aber auch dies beobachtet der Erzähler nur einen Augenblick, um dann, von Scham über seine eigene Sicherheit erfüllt, zu rufen: „Hinweg! Wir erzählen das nicht!“ Der damals vielgelesene rheinische Lyriker und Erzähler Josef Winckler schrieb 1922 unter dem Titel Der Irrgarten Gottes oder Die Komödie des Chaos einen schonungslos krassen und abgrundtief pessimistischen Abgesang auf die bluttriefende Weltgeschichte, bevor es ihm 1924 gelang, sich mit dem westfälischen Schelmenroman Der tolle Bomberg, dessen Handlung im biedermeierlichen 19. Jahrhundert spielt, aus der depressiven Stimmung der Nachkriegszeit zu befreien. Auch Carl Zuckmayer, der den Krieg von Anfang bis Ende als freiwilliger Artillerieoffizier mitgemacht hatte, bekannte, dass es ihm erst 1924 mit der Arbeit an seinem Volksstück Der fröhliche Weinberg gelang, seine Niedergeschlagenheit zu überwinden: „Zum ersten Mal seit den Kriegsjahren", so erinnert er sich in seiner Autobiographie, „erfüllte mich wieder ungetrübte Heiterkeit" 11 , und diese, so darf man wohl hinzufügen, teilte sich in solchem Maß dem Stück mit, dass es, im Dezember 1925 uraufgeführt, sofort als Auftakt einer besseren Zeit empfunden wurde und in den folgenden „Goldenen Zwanzigern“ Tag für Tag auf zumeist mehreren Bühnen gegeben wurde. Das eindrucksvollste Beispiel für die Schwierigkeit, Heiterkeit nach dem Ersten Weltkrieg zu bewahren oder wiederzugewinnen, ist wohl Hermann Hesses Roman Der Steppenwolf (1927). Dessen Held, Harry Haller, lebt ja in einer geradezu explosiven Gegnerschaft zur bürgerlichen Gesellschaft, die, wie die unglückliche Begegnung mit dem MythologieProfessor zeigt, nicht zuletzt aus seiner Gegnerschaft zum Krieg und seiner kriegskritischen Publizistik während der Kriegsjahre resultiert ${ }^{12}$. Gelassenheit und Heiterkeit sind ihm völlig abhanden gekommen, und ebenso fehlt ihm aller Humor, mit dessen Hilfe er zu einer souveränen und entspannten, zwar distanzierten, aber doch auch teilnehmenden und anerkennenden Haltung gegenüber der Welt finden könnte ${ }^{13}$. Er braucht

10. - Zu den diskreten Bezügen zum Krieg vgl. Alexander Honold, Einsatz der Dichtung. Literatur im Zeichen des Ersten Weltkriegs, Berlin, Vorwerk 8, 2015, S. 281287.

11. - Carl Zuckmayer, Als wär's ein Stück von mir, Frankfurt a.M., Fischer, 1966, S. 449.

12. - Hermann Hesse, Der Steppenwolf. Mit einem Kommentar von Heribert Kuhn, Frankfurt a.M., Suhrkamp, 1997, S. 88-98.

13. - Vgl. ebd., bes. S. 66 f. 
die Begegnung mit nicht verbiesterten Menschen, mit dem nichts als beglückenden ,Freudenmädchen“ Maria und dem ganz und gar unbekümmerten Musiker Pablo, bevor er wieder in die Lage kommt, den „Humor des Lebens“"14 zu erfassen und in einen Zustand der „Heiterkeit“" zu gelangen, die aus der Musik von Mozart und Bach zu ihm sprach ${ }^{15}$ und die zuletzt in ein helles und wildes Lachen ausbrechen möchte ${ }^{16}$. Was dieser Heiterkeit entgegensteht, wird darüber wohl nicht vergessen; nicht umsonst betont der Humor-Lehrer Pablo, dass der Humor ,,dieses Lebens“ immer auch „Galgenhumor“" sei: Bemühen um Heiterkeit im Bewusstsein der Endlichkeit und gar der drohenden Auslöschung.

Weitere Beispiele für den Verlust und die Wiedergewinnung künstlerischer Heiterkeit nach dem Ersten Weltkrieg wären leicht zu nennen, doch ist auch festzustellen, dass die Reserviertheit gegenüber dem Heiterkeitspostulat bestehen blieb. Der Weltkrieg und die Anfangs- und Endjahre der Weimarer Republik diktierten der Literatur Themen, die einer durch Heiterkeit geprägten Betrachtungs- und Darstellungsweise widerstrebten; ihre Gegenstände waren politische Kämpfe mit Mord und Totschlag, soziale Verwerfungen und ökonomische Krisen mit schlimmsten Folgen für große Teile der Bevölkerung ${ }^{17}$. Literatur sollte unter diesen Umständen nicht eine höhere, freiere Sichtweise anstreben, sondern ,,ars militans“ sein, wie Alfred Döblin im Mai 1929 in einer Sitzung der Berliner Dichterakademie verlangte ${ }^{18}$, und im sozialen und politischen Kampf als „Waffe“ dienen, wie Friedrich Wolf, Mitglied des Bundes proletarisch-revolutionärer Schriftsteller (BPRS), schon 1928 mit einem manifestartigen Artikel gefordert hatte ${ }^{19}$. Dass Kunst das Denken aus der Fatalität der Geschichte und aus den Zwängen der Politik herausführen und befreiend wirken sollte, sagte ausdrücklich allein Kurt Schwitters in seinem Anfang 1931 publizierten Manifest Ich und meine Ziele. Dort heißt es:

Kunst will nicht beeinflussen und nicht wirken, sondern befreien, vom Leben, von allen Dingen, die den Menschen belasten, wie nationale, politische und wirtschaftliche Kämpfe. [...] Ihr aber, Ihr politischen Menschen von rechts oder links, oder Ihr mittlere Sorte, oder

14. - Vgl. ebd., S. 240.

15. - Vgl. ebd., S. 175.

16. - Vgl. ebd., S. 231.

17. - Vgl. Helmuth Kiesel, Geschichte der deutschsprachigen Literatur 19181933, München, Beck, 2017, S. 207-1002.

18. - Alfred Döblin, „,Kunst ist nicht frei, sondern wirksam: ars militans“, in: Ders., Schriften zu Ästhetik, Poetik und Literatur, hrsg. von Erich Kleinschmidt, Olten und Freiburg im Breisgau, Walter, 1989, S. 245-261.

19. - Friedrich Wolf, „Kunst ist Waffe / Eine Feststellung“, in: Marxismus und Literatur. Eine Dokumentation in drei Bänden, hrsg. von Fritz J. Raddatz, Reinbek bei Hamburg, Rowohlt, 1969, hier Bd. II, S. 250-262. 
aus welchem blutigen Heerlager des Geistes Ihr kommen mögt, wenn Ihr eines Tages mal die Politik recht satt habt, oder Euch auch nur für einen Abend von Euren Strapazen ausruhen wollt, so kommt zur Kunst, zur reinen unpolitischen Kunst, die ohne Tendenz ist, nicht sozial, nicht national, nicht zeitlich gebunden, nicht modisch. Sie kann Euch erquicken und sie wird es gerne tun ${ }^{20}$.

\section{Verlust von Heiterkeit und Festhalten am Heiterkeits- postulat in der Epoche des Faschismus und Nationalsozialismus}

Der Aufstieg des Faschismus in Italien und des Nationalsozialismus in Deutschland brachte eine Fanatisierung des sozialen Klimas mit sich, die mit einer zunehmenden Entheiterung verbunden war. Thomas Mann hat diesen Vorgang in seiner 1930 erschienenen Novelle Mario und der Zauberer beschrieben ${ }^{21}$. Vermutlich geht diese Novelle auf einen Ferienaufenthalt Manns und seiner Familie im Badeort Forte dei Marmi im Jahr 1926 zurück: Man war hingefahren, um sich am Meer zu erholen, und erwartete, wie immer, eine Atmosphäre mediterraner Lebensfreude und Gelassenheit, musste aber feststellen, dass „Ärrer, Gereiztheit, Überspannung [...] von Anfang an in der Luft [lagen]"22, wie mit dem ersten Satz der Erzählung gesagt wird. Wenig später wird dies amplifiziert und nun auch als Produkt der politischen Entwicklung kenntlich gemacht:

Auf irgendeine Weise fehlte es der Atmosphäre an Unschuld, an Zwanglosigkeit; dies Publikum ,hielt auf sich“ - man wußte zunächst nicht recht, in welchem Sinn und Geist, es präsentierte Würde, stellte voneinander und vor dem Fremden Ernst und Haltung, wach aufgerichtete Ehrliebe zur Schau -, wieso? Man verstand bald, daß Politisches umging, die Idee der Nation im Spiele war ${ }^{23}$.

Von der „Größe und Würde Italiens“ ist die Rede, und bald werden sogar die kleinen Streitigkeiten der internationalen Kinderschar am

20. - Kurt Schwitters, Das literarische Werk, hrsg. von Friedhelm Lach, Bd. 5: Manifeste und kritische Prosa, München, Deutscher Taschenbuch Verlag, 2005, S. 340348, hier S. 341 und 348.

21. - Aus der Fülle von Forschungsliteratur zu Mario und der Zauberer kann hier nur auf das entsprechende, besonders einschlägige Kapitel der folgenden Untersuchung verwiesen werden: Friederike Tebben, Genie und Charisma: Herrschaftsformen und Führerfiguren im Werk Thomas Manns, Tübingen, Narr/Franke/Attempto, 2017, S. 115142.

22. - Thomas Mann, Sämtliche Erzählungen, Frankfurt a.M., Fischer, 1963, S. 523.

23. - Ebd., S. 529. 
Strand mit solch ,unheiter-spielverderberischen Redensarten“ sanktioniert 24 . Das muss nicht weiter rekapituliert werden; es reicht, festzustellen, dass Nationalismus und Faschismus zu einem deutlichen Heiterkeitsverlust geführt hat und, wie sich im weiteren Verlauf des Geschehens zeigt, Heiterkeit prinzipiell gefährdet. Denn der Auftritt des charismatisch und zugleich terroristisch wirkenden „Zauberers“ oder - eigentlich - Hypnotiseurs Cipolla, in dem man zu Recht einen Massendompteur gesehen hat, löst zwar mehrfach Lachen und „Heiterkeit“ aus ${ }^{25}$, doch ist dies eine Heiterkeit, die nicht nur Effekt von Überraschung und Staunen ist, sondern auch mit Schadenfreude verbunden ist. - Als die Novelle nach der Publikation mehrfach als Parabel auf den Faschismus gedeutet wurde, wiegelte Thomas Mann $\mathrm{ab}$, indem er am 15. April 1932 in einem Brief an einen Kritiker sagte, er wolle ihre Bedeutung - trotz ,kleine[r] politische[r] Glanzlichter und Anspielungen aktueller Art“ - ,doch lieber im Ethischen als im Politischen sehen“, ,vom Künstlerischen abgesehen“. Zugleich räumte er ein, dass „das Politische ein weiter Begriff“ sei, „der ohne scharfe

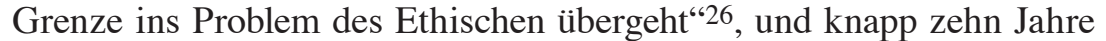
später, am 26. Juni 1941, betonte er, dass die Novelle „,entschieden einen moralisch-politischen Sinn“ habe und das Klima im Europa des heraufziehenden Faschismus zeige ${ }^{27}$. Als Hauptmerkmal dieses Klimas nannte er „menschliche Entwürdigung und Willenszwang“; dass diese mit einem Verlust an Heiterkeit verbunden waren, geht aus der Novelle deutlich hervor.

1933 wurde der Horizont für Zeitgenossen, die ihre Augen vor dem NS-Terror nicht verschlossen und die Zeichen der Zeit als Vorzeichen eines neuen Krieges zu deuten wussten, noch dunkler. Der Blick auf die Kunst änderte sich. Alfred Döblin warf in einem Brief, den er am 12. Juni 1934 aus seinem Pariser Zufluchtsort an einen befreundeten Bankier schrieb, die Frage auf, ob sein Roman Babylonische Wandrung oder Hochmut kommt vor dem Fall, den er 1932 in Berlin begonnen und dann im Exil abgeschlossen hatte, nun noch klingen könne: ,es war noch Heiterkeit und Kunst aus alter Zeit" 28 . In Briefen und Tagebüchern wird

24. - Ebd., S. 530 .

25. - Ebd., S. $536 \mathrm{ff}$.

26. - Brief vom 15.4.1932 an Bedřich Fučik, in: T. Mann, Briefe I: 1889-1936, hrsg. von Erika Mann, Frankfurt a.M., Fischer, 1979, S. 315.

27. - Brief vom 26.6.1941 an Max Flesch-Thebesius, in: Dichter über ihre Dichtungen, hrsg. von Hans Wysling und Marianne Fischer, Bd. 14/II: Thomas Mann, Teil II: 1918-1943, München, Heimeran, 1979, S. 371.

28. - A. Döblin, Briefe, hrsg. von Heinz Graber, Olten und Freiburg im Breisgau, Walter, 1970, S. 193. - Zum Aspekt der tragischen Heiterkeit der Babylonischen Wandrung vgl. H. Kiesel, Literarische Trauerarbeit. Das Exil- und Spätwerk Alfred Döblins, Tübingen, Niemeyer, 1986, S. 96-142. 
ein weiterer Verlust an Lebenszuversicht und Heiterkeit beklagt. Immer wieder wird auch die fanatische Humorlosigkeit der NS-Chargen betont, die auf die Bevölkerung abzufärben begann. Im japanischen Exil hat der Philosoph Karl Löwith in einem ersten Lebensbericht ausführlich über die „finster-energische“ Stimmung geschrieben, die sich zu Beginn des ,Dritten Reichs' ausbreitete ${ }^{29}$. Der Romanist Werner Krauss, der im November 1942 als Mitglied der Widerstandsgruppe ,Rote Kapelle“ inhaftiert wurde, schrieb im Gefängnis einen Roman, der 1946 unter dem Titel PLN - Die Passionen der halykonischen Seele erschien. „Halykonisch“ ist ein Anagramm aus ,halkyonisch", was seit mythologischen Zeiten ,heiter" und ,gelassen" bedeutet. Die „halykonische Seele“ ist indessen die unheitere, tragisch-überspannte und verbiesterte deutsche Seele, die sich in der Organisationswut des ,Dritten Reichs' auszuleben versuchte (und leider nicht nur das System der Postleitnummern/ PLN erfand).

In anderen persönlichen Aufzeichnungen und literarischen Werken aus den Jahren nach 1933 dokumentiert sich aber auch der Versuch, Heiterkeit als Lebensprinzip zu bewahren. Hermann Brochs 1933 verfasster und veröffentlichter Roman Die unbekannte Größe, in dem ein Mathematiker die irrationalen Seiten des Lebens zu erkunden sucht, enthält gleich im fünften Kapitel des ersten Teils bei der Charakterisierung der katholischen Kinder- und Krankenschwester Susanne einen wahren Hymnus auf deren „Heiterkeit“, die aus dem Gefühl der Aufgehobenheit in der „ganzen reichen Gotteswelt“ stammt, Ausdruck eines „,erwählten religiösen Daseins“ ist und sich der „Düsternis“, welche die menschliche Existenz von Anfang an umgibt, entwindet ${ }^{30}$. Auch Brochs nächstes erzählerisches Werk, der 1935 entstandene Bergroman, der später unter dem Titel Die Verzauberung erschien und wie ein gesteigertes Pendant zu Thomas Manns Mario und der Zauberer wirkt, gibt mit der Stimme des Helden und Erzählers dem Wunsch Ausdruck, inmitten des „Sumpfe[s]“ einer faschistisch fanatisierten Welt die „heilige Heiterkeit der Erde“ und die „heitere Heiligkeit der Mitte“ bewahren zu können ${ }^{31}$. Ähnliches ist bei Ernst Jünger zu sehen. Diesen hatte eine Schiffsreise im Herbst 1936 für zwei Monate aus dem nationalsozialistischen Deutschland nach Südamerika geführt. Am 15. Dezember, dem letzten

29. - Vgl. Karl Löwith, Mein Leben in Deutschland vor und nach 1933. Ein Bericht, Frankfurt a.M., Fischer, 1989, S. 7 f. und 39-42.

30. - Hermann Broch, Kommentierte Werkausgabe, hrsg. von Paul Michael Lützeler, Bd. 2: Die unbekannte Größe, Frankfurt a.M., Suhrkamp, 1977, S. 31. Weitere einschlägige Stellen: S. 34, 67, 213, 215 und 251.

31. - H. Broch, Kommentierte Werkausgabe, hrsg. von P.M. Lützeler, Bd. 3: Die Verzauberung, Frankfurt a.M., Suhrkamp, 1980, S. 321. Weitere einschlägige Stellen: 207, 241 f., 253 und 357. 
Tag der Rückfahrt, schloss er sein Reisetagebuch kurz vor der Ankunft in Hamburg mit den Sätzen:

Heut oder morgen Abfahrt nach Leisnig [dem Wohnort der Eltern], sodann nach Überlingen am Bodensee [dem neuen eigenen Wohnort]. Möge es mir vergönnt sein, an diesen sonnigen Gestaden Tage und vielleicht Jahre zu verbringen, frei, heiter, tätig - trotz allem Niedrigen, das uns umgibt ${ }^{32}$.

An den „sonnigen Gestaden“ des Bodensees entstanden dann die sichtlich von den Schrecknissen des ,Dritten Reichs“ geschwängerten Reflexionen, die 1938 in der zweiten Fassung des Abenteuerlichen Herzens erschienen. In zwei dieser Aphorismen, die vom Wunsch nach einer souveränen Haltung gegenüber der Gefahr der Vernichtung diktiert sind, spielt der Begriff der Heiterkeit eine wichtige Rolle. Unter der Überschrift „Zur Désinvolture“ beschreibt Jünger die damit gemeinte Ungezwungenheit zunächst als ,göttergleiche Überlegenheit“" von großen Herren, als Freiheit oder „Unschuld“ und „Anmut der Macht“, deklariert sie dann aber allgemeiner als eine ,,besondere Form der Heiterkeit“, die freilich erst wiederzuentdecken sei: „Die Heiterkeit gehört zu den gewaltigen Waffen, über die der Mensch verfügt - er trägt sie als göttliche Rüstung, in der er selbst die Schrecken der Vernichtung zu bestehen vermag." 33

Der unmittelbar folgende Aphorismus steht unter der Überschrift „Historia in Nuce: Der Verlorene Posten“ und handelt von Situationen, in denen die Vernichtung bereits im Gang ist und die Betroffenen sich mit ihrem Schicksal schon abgefunden haben. In solchen Momenten gewinnen alle Fristen und Handlungen, die den Betroffenen noch vergönnt sind, eine besondere Würde und endgültige Bedeutung. Zur Trauer tritt dann Heiterkeit und geht mit dieser eine ,herbstlich“ anmutende Verbindung ein ${ }^{34}$. Ebendiese Stimmung hat Jünger mit seiner elegischen Erzählung Auf den Marmorklippen, die im Frühjahr 1939 nach sechs Jahren NS-Herrschaft und in der sicheren Erwartung eines neuen Krieges verfasste, reproduziert. Gleich der erste Satz charakterisiert den Erzähler als jemanden, der durch die Erinnerung an ein vormals kultiviertes und glückliches Leben und an dessen Zerstörung durch einen atavistischen Usurpator in eine ,wilde Schwermut“"35

32. - Ernst Jünger, Sämtliche Werke, Bd. 6: Reisetagebücher, Stuttgart, KlettCotta, 1982, S. 183 (Atlantische Fahrt).

33. - E. Jünger, Sämtliche Werke, Bd. 9: Das abenteuerliche Herz, Stuttgart, Klett-Cotta, 1979, S. $261 \mathrm{f}$.

34. - Ebd., S. 264.

35. - E. Jünger, Sämtliche Werke, Bd. 15: Erzählungen, Stuttgart, Klett-Cotta, 1978, S. 249. Zum geschichtlichen Hintergrund und zum Charakter des Werks vgl. E. 
gebracht wird und diese offensichtlich durch das Niederschreiben seiner Geschichte und die Beschwörung rettender Perspektiven zu bändigen versucht. Auch den Charakter dieses Werks kann man - mit Jüngers Formulierung aus dem Abenteuerlichen Herzen - als eine „herbstliche Mischung aus Trauer und Heiterkeit" bezeichnen. Die Heiterkeit, die in diesem Werk unter tödlicher Bedrohung steht, erscheint in zweifacher Form. Zum einen zeigt sie sich, wie Harald Weinrich verdeutlicht hat, in der Heiterkeit einiger Figuren: „beim Erzähler als ästhetische Heiterkeit des Literaten und Künstlers, bei seinem Sohn Erio als vitale Heiterkeit, bei dem Mönch und Bibliothekar Lampros als die etwas stubenbleiche Heiterkeit des Gelehrten und schließlich als adelig-edle Heiterkeit des Fürsten [Sunmyra]“36. Zum andern zeigt sich Heiterkeit aber auch in den überlegen sich gebenden Reflexionen des Erzählers und in der sprachlichen Gestaltung, die durch ihren gefassten Duktus, ihr elegisches Pathos, ihre Sentenzhaftigkeit und ihr preziöses Vokabular sowohl ergreifend als auch tröstlich wirken mag, sofern sich der Leser nicht gegen diesen suggestiven und oft als kitschig kritisierten ,sound sperrt. Heiterkeit als Lebenshaltung war für Jünger in diesen Jahren indessen nicht leicht zu bewahren. Zwei Porträts, die von den mit Jünger befreundeten Malern A. Paul Weber und Rudolf Schlichter 1936 und 1937 geschaffen wurden, zeigen Jünger nicht etwa mit heiterer Miene und in entspannter Haltung, sondern - bei A. Paul Weber - in mühsam beherrschter Aufgew ühltheit und - bei Rudolf Schlichter - mit gefasstem, aber ernstem Blick, der in eine offensichtlich bedrohliche Ferne geht ${ }^{37}$. Im Ersten Pariser Tagebuch über den Dienst als Besatzungsoffizier wird dann - wohl unter dem Eindruck der Geiselerschießungen und der zunehmenden Aktivitäten des Sicherheitsdienstes (SD) - „Tristitia“ zu einem mehrfach gebrauchten Kennwort der seelischen Befindlichkeit ${ }^{38}$.

\section{Reklamation von Heiterkeit in zeitgenössischen Epochenreflexionen}

Auch für den im Exil befindlichen Thomas Mann wurde es schwer, am Prinzip der Heiterkeit festzuhalten. Nach dem Abschluss der JosephTetralogie im Januar 1943 stellte sich für ihn die Frage, welchem Werk

Jünger, Aufden Marmorklippen. Mit Materialien zu Entstehung, Rezeption und Debatte, hrsg. von H. Kiesel, Stuttgart, Klett-Cotta, 2017, S. 303-387.

36. - H. Weinrich, Kleine Literaturgeschichte der Heiterkeit, a.a.O., S. 48.

37. - Reproduktionen in der zuletzt genannten Ausgabe der Marmorklippen, S. 147 und 154.

38. - Beginnend mit dem 29. April 1941: vgl. E. Jünger, Sämtliche Werke, Bd. 2: Strahlungen I, Stuttgart, Klett-Cotta, 1979, S. 236, 307 („Traurigkeit“), 320, 399 („Böse Melancholie“), 402 („,betrübte Nacht“), 404 („Nachts starke Attacken: La frousse“), 405 (,Schlechte Nacht. La frousse“). 
er sich nun widmen sollte. In Frage kamen zwei ältere Pläne: ein bereits 1901 knapp skizziertes Faust-Buch und die 1905 geplante und ab 1910 mehrfach in Arbeit genommene Hochstaplergeschichte, die 1954 unter dem Titel Bekenntnisse des Hochstaplers Felix Krull erschien ${ }^{39}$. Unter dem Eindruck des Krieges und der immer schrecklicher werdenden Nachrichten über Deutschland entschloss Mann sich, den KrullRoman zurückzustellen und mit dem Doktor Faustus ein Buch über sein unglückseliges „Vaterland“ (so das letzte Wort des Romans) zu schreiben. Bekanntlich rekonstruiert dieser von 1943 bis 1947 entstandene Roman, indem er ,das Leben des deutschen Tonsetzers Adrian Leverkühn“ (so der Untertitel) „,von einem Freunde“ erzählen lässt, die geistige Entwicklung Deutschlands ${ }^{40}$, die aus der wilhelminischen Zeit in den Präfaschismus führte, das ,Dritte Reich“ ermöglichte und in der Katastrophe der letzten Kriegsjahre ihren Abschluss fand. Für diese unerfreuliche Aufgabe erkor sich Mann einen gutbürgerlichen und sensiblen, aber auch sehr kontrollierten und an Schicklichkeit festhaltenden Altphilologen zum Erzähler und gab ihm, um den Charakter sowohl der Person als auch seiner Erzählweise zu profilieren, den Namen Serenus (der Heitere) Zeitblom (wohl Blume oder Blüte der Zeit). Dessen Rapport setzt mit dem 23. Mai 1943 ein und endet mit dem Ausgang des Krieges im Frühjahr 1945, als das ganze Verhängnis in allen seinen Zusammenhängen und Dimensionen sichtbar wird. Die Verschlingung von Kultur und Barbarei, von künstlerischer Progression und ,,intentioneller Re-Barbarisierung" oder Dehumanisierung wird aufgewiesen ${ }^{41}$. Das ,Dritte Reich“ erscheint dem Erzähler als „Gefängnis“ und „Narrenhaus“42. Die Zerstörung der deutschen Städte durch die alliierten Bomber wird mehrfach erwähnt 43 . Von ,dickwandigen“ und „schalldichten“ Folterkellern ist die Rede ${ }^{44}$, und endlich auch von dem Grauen, das in den befreiten Konzentrationslagern ansichtig wurde ${ }^{45}$. Zugleich wird Leverkühns musikalisches Schaffen geschildert, das die deutsche Unheilsgeschichte reflektiert und in „Dr. Fausti Weheklag“ seinen Abschluss findet: in einem „Monstre-Werk der Klage“ 46 , einem „riesenhafte[n] Lamento“ von „fünf Viertelstunden“ Dauer ${ }^{47}$, das einer „Zurücknahme“ von Beethovens neunter Symphonie und speziell ihres

39. - Die folgenden Seitenangaben beziehen sich auf T. Mann, Doktor Faustus. Die Entstehung des Doktor Faustus, Frankfurt a.M., Fischer, 1981, hier S. 691 und 693.

40. - Konzentriert ebd., S. 400 (Kap. XXX) und 450-454 (Kap. XXXIII).

41. - Ebd., S 82 (Kap. VIII), 378 (Kap. XXVIII), 491 und 495 f. (Kap. XXXIV).

42. - Ebd., S. 44 (Kap. V) und 336 (Kap. XXVI).

43. - Ebd., S. 231 (Kap. XXI), 335 (Kap. XXVI) und 576 (Kap. XLI).

44. - Ebd., S. 327 (Kap. XXV) und 637 f. (Kap XLVI).

45. - Ebd.

46. - Ebd., S. 644 (Kap. XLVI).

47. - Ebd., S. 645. 
letzten Satzes mit dem Jubel von Schillers „Lied an die Freude“ gleichkommt ${ }^{48}$ und allenfalls in jenem „hohe[n] g eines Cellos“, mit dem das Werk leise ausklingt, an die Möglichkeit von Hoffnung und Heilung denken lässt ${ }^{49}$.

Thomas Mann beschrieb dieses Werk detailliert und eindringlich, ließ aber seinen Roman keineswegs zu einem vergleichbar konsequenten Werk der Klage werden, sondern hielt am Prinzip der Heiterkeit fest und bemühte sich, wie er in der nachfolgenden Schrift Die Entstehung des Doktor Faustus wörtlich sagte, ,um eine gewisse Durchheiterung des düsteren Stoffes" ${ }^{\prime 50}$. Als Mittel dienten die Namensgebung, die Thomas Mann eigens erwähnt ${ }^{51}$, und die Gestaltung der Figuren, von denen manche komische und erheiternde Züge erhielten; bei den zumeist gemeinsam auftretenden Damen Meta Nackedey, Klavierlehrerin, und Kunigunde Rosenstiel, Mitinhaberin einer Wurstdarmfabrik, wirken beide Mittel zusammen ${ }^{52}$. Die „Durchheiterung“ des wahrhaft und erdrückend ,düsteren“ Stoffes brauchte allerdings eine nicht ganz unproblematische Ermöglichung und sozusagen negative Flankierung. Diese besteht - wie schon im Zauberberg - im Verzicht auf die Darstellung der Sphären der Vernichtung. Vom Inferno der Bombennächte, vom Leben ,im Schutt unserer Städte“ unter Ratten, die ,,von Leichen fett“ waren, berichtet Zeitblom nur in wenigen Worten ${ }^{53}$, desgleichen von den erschütternden Bildern, welche die Bevölkerung von Weimar zu sehen bekam, als ein amerikanischer General sie zwang, das Konzentrationslager Buchenwald aufzusuchen und sich dem Anblick der Baracken, Folterstätten und Verbrennungsöfen zu stellen ${ }^{54}$. Dieser Verzicht geschieht selbstverständlich nicht unreflektiert; vielmehr heißt

48. - Ebd., S. 634 (Kap. XLV) und 649 (Kap. XLVI).

49. - Ebd. S. 651.

50. - Ebd., S. 700 - Vgl. zu diesem Aspekt insbesondere: H. Kiesel, „Thomas Manns Doktor Faustus. Reklamation der Heiterkeit“, Deutsche Vierteljahrsschrift für Literaturwissenschaft und Geistesgeschichte, 64/1990, S. 726-743, sowie Dieter Borchmeyer, „Musik im Zeichen Saturns. Melancholie und Heiterkeit in Thomas Manns Doktor Faustus“, Thomas Mann Jahrbuch, 7/1994, S. 123-167, und Sibylle SchulzeBerge, Heiterkeit im Exil. Ein ästhetisches Prinzip bei Thomas Mann, Würzburg, Königshausen \& Neumann, 2006, bes. S. 169-234.

51. - T. Mann, Die Entstehung des Doktor Faustus, a.a.O., S. 730 (Kap. VII) und zuvor 705 (Kap. V).

52. - Dass Namenskomik eine letztmögliche Quelle von Heiterkeit ist, betont Odo Marquard, „Exile der Heiterkeit“, in: Wolfgang Preisendanz/Rainer Warning (Hrsg.), Das Komische, München, Fink, 1976, S. 133-151.

53. - T. Mann, Doktor Faustus, a.a.O., S. 576 (Kap. XLI).

54. - Ebd., S. 637 (Kap. XLVI) und S. 757 (Kap. IX der Entstehung). - Thomas Manns eigene Betroffenheit zeigt sich in seinem Artikel „Die Lager“, der am 18. Mai 1945 in der Bayrischen Landeszeitung erschien. In: Thomas Mann, Politische Schriften und Reden, hrsg. von Hans Bürgin, Frankfurt a. M., Fischer, 1968, hier Bd. 3, S. 160 f. 
es in dem Gespräch, das Adrian mit dem Teufel führt, dass das, was in den „schalldichten“ Kellern geschehe, ,abseits und außerhalb der Sprache“ liege 55 . Das sagt zwar der Teufel, aber bekanntlich hat dieser im Doktor Faustus eine beträchtliche ästhetische Kompetenz.

Auch in anderen Werken der letzten Weltkriegs- und ersten Nachkriegsjahre wird Heiterkeit thematisiert, in Frage gestellt oder als Lebens- und Kunstprinzip beschworen. In Brochs Vergil-Roman, entstanden zwischen 1936 und 1944, sehnt sich der sterbensmüde, aus Athen nach Rom zurückkehrende Dichter, wie immer wieder angedeutet wird, nach Heiterkeit, doch geben die Verhältnisse, die er vorfindet, ,wenig Anlaß“ dazu ${ }^{56}$. In Hermann Hesses Glasperlenspiel, 1943 erschienen, ist am Ende des einführenden Kapitels von „übermenschlichem Lachen“ und „unsterblicher Heiterkeit“ die Rede, wovon die klassische Musik kündet und wovon auch alle menschlichen „Glasperlenspiele“ und alles menschliche „Leben, Tun und Leiden“" erfüllt sein soll ${ }^{57}$. Ähnliches findet sich, vielleicht durch das Glasperlenspiel angeregt, am Ende von Hermann Kasacks surrealistisch geartetem Roman Die Stadt hinter dem Strom, der zwischen 1942 und 1946 entstand. Der Protagonist dieses Romans wird dort unversehens in ein schattenhaftes „Reich des Todes“58 versetzt, in ein „Land ohne Freude“"59. Heiterkeit gibt es allein in der zuletzt ansichtigen Sphäre der „dreiunddreißig Weltenwächter“" von Heraklit und Lao Tse über Augustinus und Montaigne bis zu Pascal und Kierkegaard60; aber diese Heiterkeit artikuliert sich in einem ,metaphysische[n] Gelächter“61, das den Protagonisten des Romans, der noch unter dem Eindruck erschütternder Bilder der Vernichtung steht, mit Zorn erfüllt und rebellieren lässt - bis er endlich bemerkt, dass nach der Entladung von Hass und Barbarei, die er zu beobachten hatte, ,Tröstung und Wohltun sich unter den Menschen wieder kräftiger regten" 62 .

55. - Ebd., S. 327 (Kap. XXV).

56. - H. Broch, Kommentierte Werkausgabe, hrsg. von P. M. Lützeler, Bd. 4: Der Tod des Vergil, Frankfurt a.M., Suhrkamp, 1976, S. 39.

57. - Hermann Hesse, Das Glasperlenspiel, Frankfurt a.M., Suhrkamp, 2002, S. 42. - Zur fundamentalen Bedeutung der Heiterkeit für Josef Knechts späteres Leben und mithin den ganzen Roman vgl. Béatrice Poulain, Poétique de l'allégresse: initiation à la Heiterkeit dans l'œuvre en prose d'Hermann Hesse, http://www.e-sorbonne.fr/ theses/poetique-1-allegresse-initiation-heiterkeit-l-oeuvre-prose-d-hermann-hesse.

58. - Hermann Kasack, Die Stadt hinter dem Strom, Frankfurt a.M., Suhrkamp, 1988, S. 258 (und S. 396: „Totenstadt“).

59. - Ebd., S. 421.

60. - Ebd., S. $408 \mathrm{f}$.

61. - Ebd., S. 405.

62. - Ebd., S. 410. Vergleichend behandelt werden Doktor Faustus, Das Glasperlenspiel und Die Stadt hinter dem Strom von Petra Kiedaisch, Ist die Kunst noch heiter? Theorie, Problematik und Gestaltung der Heiterkeit in der deutschsprachigen 
Noch mehr als Kasack hegten andere Autoren die Vorstellung, dass die Weltgeschichte nach der Niederschlagung des Nationalsozialismus trotz aller vergangenen Greuel und verbliebenen Probleme eine positive Wendung nehmen könne. Thomas Mann schrieb am 1. Januar 1947 an Karl Kerényi unter Bezugnahme auf die „Weheklag“ seines Faustus-Romans:

Nun, Klage ist ja ein recht aktueller Ausdrucksgehalt, finden Sie nicht? Es sieht böse aus. Meine Nachrichten aus Deutschland zumal sind gar so hoffnungslos. Freilich glaube ich im Grunde, daß, alles in allem, die Menschheit doch, trotz allem gegenteiligen Anschein, ein gutes Stück vorwärts gestoßen worden ist. Auch ist sie eine zähe Katze. Selbst die A-Bombe macht mich nicht ernstlich bange ihretwegen. Erweist sie sich nicht als zäh in uns selbst? Welch sonderbarer Leichtsinn, oder welche Vertrauensseligkeit, daß wir noch immer Werke schaffen! Für wen? Für welche Zukunft? Und doch, ein Werk, und sei es eines der Verzweiflung, kann immer nur den Optimismus, den Glauben ans Leben zur letzten Substanz haben - wie es ja mit der Verzweiflung eine besondere Sache ist: sie trägt die Transzendenz zur Hoffnung schon in sich selbst ${ }^{63}$.

Diese These, die wörtlich das Ende der Beschreibung der „Weheklag“ im Doktor Faustus zitiert, ermöglichte es Thomas Mann, auch nach dem Zweiten Weltkrieg und nach dem Holocaust an jener Art von literarischem Schaffen festzuhalten, die er 1936 in einer Rede als seinen „Lebensinhalt" bezeichnet hatte und die er 1953 mit der Publikation dieser Rede erneut beschwor: an dem "heiteren und strengen Dienst“ am „Wort"64. Und als wolle er die heitere Seite dieses Dienstes betonen, wandte er sich nun, 1950, wieder der Schelmengeschichte des Felix Krull zu, die er 1943 zugunsten des ,düsteren“ Deutschlandbuches zurückgestellt hatte.

\section{Fragwürdige Behauptung einer, ,heilen Welt“ und Gegenreden}

Ähnlich wie Thomas Mann blickte auch Werner Bergengruen, der dem Nationalsozialismus ablehnend gegenüber gestanden hatte und wegen der jüdischen Herkunft seiner Frau ausgegrenzt worden war, nach 1945 versöhnlich auf die Welt und optimistisch in die Zukunft. 1950 publizierte er unter dem Titel Die heile Welt einen Band mit

Literatur nach 1945, Tübingen, Niemeyer, 1996, S. 33-73.

63. - Thomas Mann - Karl Kerényi: Gespräch in Briefen, Zürich, Rhein-Verlag, 1960, S. 146.

64. - T. Mann, „Humaniora und Humanismus“, in: Ders., Politische Schriften und Reden, a.a.O., Bd. 2, S. 324-331, hier 329. 
rund 150 Gedichten, die eine einzige Feier der grundsätzlich und von neuem heilen Welt darstellen. Wohl erinnern einige an die Gefallenen des Weltkriegs, und wohl ist im Einleitungsgedicht von stürmischen Winden die Rede, die von Zeit zu Zeit über die Erde brausen und Menschen mit sich reißen; aber die meisten anderen Gedichte sind Preis der Elemente, der Natur mit ihren Landschaften, Pflanzen und Tieren, kurz: der Schöpfung und des Schöpfers. Der Mensch wird trotz der jüngsten geschichtlichen Verfehlungen und Verbrechen nicht ausgenommen, denn „Schrecknisse“, so heißt es im Gedicht „Ruhm des Menschen und seiner Zukunft“, ,,sind die Ammen der Zukunft“"65. Von Heiterkeit ist in all den Gedichten nicht ausdrücklich die Rede. Aber Inhalt und poetische Faktur, Lobpreisungen und eingängige Reimtechnik machen den Band zu einem unerwarteten und fast umwerfenden Beispiel von Behaglichkeit und Heiterkeit. Dass Bergengruen dies bewusst anstrebte, ist als gewiss zu betrachten. In seinen Aufzeichnungen aus jenen Jahren warf er den zeitgenössischen „Tendenzdichtern“ vor, sie wollten die Welt verbessern, was er indessen nicht nur als vergebliches Bemühen, sondern als Anmaßung aus einem dreifachen Mangel betrachtete: „Wer es [i.e. das Verbessern der Welt] will, hat weder Ehrfurcht noch Demut noch Heiterkeit, und gerade diese drei sind uns am nötigsten. Wo sie sind, ist die Welt von selber gebessert.“66

Mit dem Festhalten am Lebens- und Kunstprinzip waren indessen nicht alle Autoren einverstanden. Gustav René Hocke wandte sich im November 1946 mit einem Artikel im Ruf gegen die „Kalligraphie“ der Autoren, die - wie Bergengruen - in gepflegter Diktion sozusagen an den fürchterlichen Gegebenheiten der NS-Zeit vorbeischrieben ${ }^{67}$. Wolfgang Borchert proklamierte 1947 unter dem Titel Das ist unser Manifest eine neue Poetik, deren Kennzeichen nicht Harmonie, sondern Dissonanz, nicht Kalligraphie, sondern Kakophonie, nicht Heiterkeit, sondern schonungsloser Realismus sein sollten ${ }^{68}$, und das eindrucksvollste Lachen, von dem in seinen Erzählungen die Rede ist, ist das grelle und „grauenhafte“ Lachen des Wahnsinns, in den der kanadische Bomberpilot Billbrook zu fallen droht, als ihm unmittelbar nach dem Krieg beim Gang durch den zerbombten Stadtteil Billbrook deutlich

65. - Werner Bergengruen, Die heile Welt. Gedichte, München, Nymphenburger, 1950, S. 26.

66. - Compendium Bergengruenianum, unpubliziertes Manuskript), S. 1101 (Deutsches Literaturarchiv Marbach.

67. - Gustav René Hocke, „Deutsche Kalligraphie oder: Glanz und Elend der modernen Literatur", in: Der Ruf. Eine deutsche Nachkriegszeitschrift, hrsg. von Hans Schwab-Felisch, München, Deutscher Taschenbuch Verlag, 1962, S. 203-208.

68. - Wolfgang Borchert, „Das ist unser Manifest“, in: Ders., Das Gesamtwerk. Mit einem biographischen Nachwort von Bernhard Meyer-Marwitz, Hamburg, Rowohlt, 1949, S. 308-315. 
wird, woran er mitgewirkt hat ${ }^{69}$. Die Poetik der Heiterkeit wird hier wie in anderen Erzählungen Borcherts und in seinem Drama Draußen vor der Tür durch eine Poetik des schonungslosen Realismus in grotesker Zuspitzung und sarkastischer Ausformulierung ersetzt ${ }^{70}$.

\section{Debatte über die Heiterkeit der Kunst}

Zehn Jahre später hat auch Paul Celan mit seiner Antwort auf eine Umfrage der Pariser Librairie Flinker festgestellt, die deutsche Lyrik müsse, da sie „Düsterstes im Gedächtnis“ und „Fragwürdigstes um sich her" habe, eine ,,grauere“ Sprache“ pflegen und dürfe „nichts mehr mit jenem ,Wohlklang“ gemein“ haben, ,der noch mit und neben dem Furchtbarsten mehr oder minder unbekümmert einhertönte" ${ }^{671}$. Die poetische Umsetzung des Postulats der ,grauere[n] Sprache“ ist auch in Celans ganzem Werk zu beobachten.

Der von Celan kritisierte „Wohlklang“ eines Teils der deutschen Nachkriegslyrik, der in Bergengruens Gedichtband Die heile Welt in reinster Form zu vernehmen war, gab Theodor W. Adorno Anlass, nach dem Verhältnis von Poesie und jüngerer Geschichte mit ihrem alle vorherigen Verfehlungen überbietenden Kulminationspunkt Holocaust $\mathrm{zu}$ fragen ${ }^{72}$. Seine Reflexionen führten bekanntlich zu der vielberufenen These, dass es „barbarisch“ und mithin „unmöglich“ sei, „nach Auschwitz ein Gedicht zu schreiben"73. Diese These, die ganz am Ende des 1949 verfassten und 1951 publizierten Essays Kulturkritik und Gesellschaft steht und dessen Quintessenz bildet, hat Adorno, veranlasst auch durch mancherlei ästhetisch-theoretische Einwände und poetische Widerlegungsversuche, in zwei weiteren Essays - Jene zwanziger Jahre und Engagement (beide 1962) - zunächst bekräftigt ${ }^{74}$, in seiner Negativen Dialektik (1966) aber durch die Feststellung revoziert, dass „das perennierende Leiden“ der Menschen selbstverständlich ein

69. - W. Borchert, Billbrook, ebd., S. 75-93, hier S. $87 \mathrm{f}$.

70. - Vgl. P. Kiedaisch, Ist die Kunst noch heiter?, a.a.O., S. 73-77.

71. - Paul Celan, Gesammelte Werke in fünf Bänden, hrsg. von Beda Allemann und Stefan Reichert unter Mitwirkung von Rolf Bücher, hier Bd. 3: Gedichte III, Prosa, Reden, Frankfurt a.M., Suhrkamp, 1986, S. 167.

72. - Auf Bergengruens Heile Welt nahm Adorno ausdrücklich Bezug, in schriftlicher Form allerdings erst 1964 in dem großen Essay „Jargon der Eigentlichkeit“. In: Theodor W. Adorno, Gesammelte Schriften, hrsg. von Rolf Tiedemann, Bd. 6: Negative Dialektik. Jargon der Eigentlichkeit, Frankfurt a.M., Suhrkamp, 1973, S. 413526, hier. S. $528 \mathrm{f}$.

73. - Alle Texte zu diesem Thema sind zusammengestellt und leicht greifbar in Lyrik nach Auschwitz? Adorno und die Dichter, hrsg. von Petra Kiedaisch, Stuttgart, Reclam, 1995, hier S. 49.

74. - Ebd., S. 53 f. 
Anrecht auf Ausdruck habe, auch im Gedicht ${ }^{75}$. Zugleich warf Adorno aber die Frage auf, ob unter diesen Umständen - also im Bewusstsein sowohl des geschichtlichen als auch des fortdauernden Leidens der Menschen - Kunst noch heiter sein könne und dürfe. Seine Antwort, 1967 in dem äußerst prägnanten Artikel Ist die Kunst heiter? publiziert, ist ein entschiedenes und umfassendes „Nein“: „Kunst, die anders als reflektiert gar nicht mehr möglich ist, muß von sich aus auf Heiterkeit verzichten."76 Dafür nennt Adorno zwei Gründe: zum einen die schamlos kalkulierte Heiterkeit der von der Kulturindustrie in Dienst genommenen und pervertierten Kunst, zum andern - „und vor allem“ - ,was jüngst geschah“, also die Verbrechen des „Faschismus“ und speziell der Holocaust. Mit dem „Faschismus“ (gemeint ist der Nationalsozialismus) erreichte der Terror eine Qualität, die - anders als in den Zeiten davor - kein „Lachen“ und mithin keine komödienhafte, humoristische und Heiterkeit vermittelnde Darstellung mehr erlaubte: „Die blutige Realität [der NS-Zeit] war nicht jener Geist oder Ungeist, dessen der Geist zu spotten vermöchte. Das waren noch gute Zeiten, mit Schlupfwinkeln und Schlamperei mitten im System des Grauens, als Haček den Schwejk schrieb." 77 Diese Zeiten, die es der Kunst noch erlaubten, sichtbar dem Heiterkeitspostulat zu folgen, sind vorbei. Die danach zu beobachtende exorbitante Progression des staatlich organisierten und mit modernsten Mitteln realisierten Terrors belegt Kunst mit einem Heiterkeitsverbot.

Die zuletzt genannten Essays fielen in eine Zeit, in welcher der Holocaust zunehmend in Erinnerung gerufen und öffentlich thematisiert wurde ${ }^{78}$. Ein paar Daten mögen dies illustrieren: 1964/65 fand in Frankfurt am Main der Auschwitz-Prozess statt, der über längere Zeit hin auf bedrückende Weise öffentlich konkret machte, was Auschwitz bedeutete. 1965 wurde Peter Weiss' dokumentarisches Drama Die Ermittlung, das auf den Prozess-Protokollen beruht, simultan auf fünfzehn Bühnen der Bundesrepublik und der DDR uraufgeführt und bewirkte eine starke Intensivierung der Auseinandersetzung mit der NS-Vergangenheit und ihrer mangelnden Aufarbeitung. Zugleich lief von 1964 bis 1966 die Verjährungsdebatte, die zu dem Ergebnis führte, dass die bis dahin nur halbherzig betriebene Verfolgung der Täter nicht eingestellt wurde. 1967 erschien die von den Psychologen Alexander und Margarete Mitscherlich verfasste Abhandlung Die Unfähigkeit zu trauern, die - pointiert gesagt - dem Heiterkeitspostulat ein Trauer-

75. - Ebd., S. 57.

76. - Ebd., S. 68.

77. - Ebd., S. 69.

78. - Hierzu H. Kiesel, ,"So ist unser Gedächtnis jetzt angefüllt mit Furchtbarem”“", in: Historikerstreit und politische Bildung, hrsg. Klaus Oesterle/Siegfried Schiele, Stuttgart, Metzler, 1989, S. 42-94. 
postulat entgegen - oder wenigstens zur Seite stellte und Trauer zum Modus der deutschen Geschichtserinnerung erhob. Welche Rolle Heiterkeit im Lebensgefühl und in der Kunst noch spielen durfte, wurde nun zum Gegenstand eines allgemeineren Nachdenkens und führte dazu, dass die Deutsche Akademie für Sprache und Dichtung 1972 ihre Preisaufgabe der Frage widmete, ob ,unserer Literatur die Heiterkeit vergangen" sei. Der Gewinner, Peter Eichhorn, gab seiner Antwort den Titel Kritik der Heiterkeit ${ }^{79}$, rekapitulierte die Heiterkeitsdebatte bis zu Adorno und registrierte umsichtig die Erscheinungsformen von Heiterkeit in der Nachkriegsliteratur mit all ihren Modifikationen, Einfärbungen, Brüchen und Schwundstufen. Dass der Kunst Heiterkeit ganz vergangen sei, konnte er nicht bestätigen, und dass sich die Kunst der Heiterkeit enthalten solle, wollte er nicht gelten lassen; vielmehr sah er in der Fähigkeit zur „Distanzierung“ eine bleibende Quelle von Heiterkeit, wenn auch einer vielfach gebrochenen, abgeschwächten, leiseren.

\section{Heiterkeit und vermiedene Heiterkeit in der deutschen Nachkriegsliteratur}

Eichhorns Befunde wurden zwanzig Jahre später durch die Dissertation von Petra Kiedaisch über „Theorie, Problematik und Gestaltung der Heiterkeit in der deutschsprachigen Literatur nach 1945“ durch Einbeziehung weiterer und jüngerer Texte erweitert und analytisch vertieft ${ }^{80}$. Mit Kiedaisch kann man in der Nachkriegszeit mehrere, wenn auch nicht scharf trennbare Phasen der Entfaltung und Modifikation von Heiterkeit unterscheiden: Ältere Autoren wie Hermann Hesse, Thomas Mann, Ernst Jünger und Hermann Kasack halten am älteren Heiterkeitskonzept fest und verbinden damit „Freiheit und Souveränität des Dichters gegenüber seinem Stoff, Humor und Ironie, Klarheit, Transparenz, Erhebung"81. Bei jüngeren Autoren wie Günter Eich, Heinrich Böll, Wolfgang Koeppen und Marie Luise Kaschnitz nehmen die „Komplemente des Heiteren“, nämlich „Melancholie und Ernst“, derart überhand, dass Heiterkeit kaum mehr aufkommen kann und als verlorene Seins- und Schreibmöglichkeit elegisch beklagt wird ${ }^{82}$. Eine weitere Phase von Heiterkeit verdankt sich der experimentellen Literatur von Eugen Gomringer und anderen, die sich der moderner und optimistischer werdenden westlichen Nachkriegswelt widmet und

79. - Peter Eichhorn, Kritik der Heiterkeit, Heidelberg, Lambert Schneider, 1973.

80. - Das Folgende ist eine auf die wichtigsten Stichworte reduzierte Zusammenfassung von P. Kiedaisch, Ist die Kunst noch heiter?, a.a.O., S. 249-260.

81. - Ebd., S. 249.

82. - Ebd., S. 252. 
durch ,intellektuelle Distanz, Freiheit, Überlegenheit, Spiel, Phantasie, Leichtigkeit und Freude“ gekennzeichnet ist ${ }^{83}$. Nach 1967 zeichnen sich zwei hauptsächliche Spielarten von Heiterkeit ab ${ }^{84}$ : Die eine realisiert sich in Ironie, Parodie, Artistik und Witz, bewegt sich aber immer am Rand der Klage oder des Protests und verzichtet auf versöhnliche Gesten. Die andere, die sich in zunehmendem Maß bei Peter Handke findet, tendiert - ohne das geschichtliche Unheil zu verleugnen - zu einer neuen Offenheit und Freiheit gegenüber der Welt und dem Leben, zur Gewinnung neuer Menschlichkeit und Gelassenheit. In der Postmoderne der 1980er und 1990er Jahre nimmt diese Tendenz überhand und werden die Komplemente der Heiterkeit, Ernst und Trauer, weitgehend abgestreift.

Adornos Postulat, dass Kunst unter den gegebenen geschichtlichen Bedingungen auf Heiterkeit verzichten müsse, konnte keine Realisierung finden. Der Grund dafür lag aber nicht etwa in der mangelnden Bereitschaft der Autoren, in ihrem Schreiben dem ,perennierende[n] Leiden“ der Menschen thematisch und stilistisch Rechnung zu tragen. Kunst selber scheint diesem Postulat zu widersprechen, insofern allein schon in der Distanzierungs- und Gestaltungsleistung, aus der sie resultiert, der Keim der Erheiterung liegt, so sachte diese auch bleiben mag. Adornos Postulat ist mithin nie voll, sondern nur annäherungsweise realisierbar. Es gibt allerdings Werke, die in dieser Hinsicht so weit vordringen, dass sich statt Heiterkeit Schmerz und trostlose Trauer einstellen. Neben Paul Celans Gedichten sei hier Peter Weiss' dreiteiliger Roman Die Ästhetik des Widerstands (1975-81) genannt. Er rekapituliert, abbreviatorisch gesagt, die NS-Zeit, handelt speziell von den Problemen des Widerstands und reflektiert die Bedeutung der Kunst für die Arbeiterbewegung und den Widerstand. Er beginnt mit der Schilderung des Pergamon-Altars, und das heißt: mit Bildern einer grauenhaften Metzelei, die nicht mit den Augen von Siegern, sondern von Unterdrüickten und potentiellen Opfern gesehen werden. Das setzt sich fort in weiteren Betrachtungen von Kunstwerken, die zum Teil sehr detailliert geschildert und erörtert werden: Dantes Divina Commedia, Kafkas Schloss, Picassos Guernica, Géricaults Floß der Medusa, Delacroix' Die Freiheit führt das Volk, Goyas Erschießung der Aufständischen und so weiter. Es sind dichterische oder malerische Bilder aus der „Geschichte der Menschheit“, die eine einzige "Geschichte des Mordens“ ist ${ }^{85}$. Sie sind Zeugnisse von künstlerischer „Hellsichtigkeit und Empörung“, aber auch von

83. - Ebd.

84. - Ebd., S. $255 \mathrm{ff}$.

85. - Peter Weiss, Die Ästhetik des Widerstands, Frankfurt a.M., Suhrkamp, 1983, Teil III, S. 87. 
„Wankelmut und Verblendung“86. Allemal sind sie aber „Wegzeichen im Gewirr der historischen Linien" ${ }^{87}$ und zugleich in der gegenwärtigen Situation des Leidens und Kämpfens. Wie die Produktion von künstlerischen Werken ist auch die Auseinandersetzung mit ihnen eine Stärkung gegen die destruktiven Gewalten und die drohende Vernichtung88. Ihre Betrachtung bewirkt jedoch nicht Tröstung oder gar Aufheiterung, sondern Trauer ${ }^{89}$. Momente des manifest Heiteren oder Humoristischen fehlen in der Ästhetik des Widerstands völlig, abgesehen von der einen Stelle, an der referiert wird, dass die Menschen auf dem Floß der "Medusa“ „mitten unter den Schrecknissen“ noch „zum Scherzen“ fähig waren, als nämlich einer der fast Verzweifelten wünschte, die Brigg ,Argus, auf deren Auftauchen sie hofften, möge doch auch „Argusaugen“" haben ${ }^{90}$. Auch die erzählerische Gestaltung mit ihren langen, nicht untergliederten Kapiteln voller bedrückender Informationen und Reflexionen, die in gedrängten Sätzen und einer nüchtern und zugleich pathetisch wirkenden Sprache wie zwanghaft dahinströmen, wollen kein Aufatmen, keine Entspannung und keine mildernde Distanzierung zulassen. Statt um „Durchheiterung“ hat Weiss sich um radikale Entheiterung bemüht. Seine Darstellung der Hinrichtung der Mitglieder der ,Roten Kapelle‘ gegen Ende des dritten und letzten Teils lässt den Leser trostlos zurück.

86. - Ebd., Teil I, S. 349.

87. - Ebd., S. 341.

88. - Ebd., Teil II, S. 177 und 213.

89. - Ebd., S. 28.

90. - Ebd., S. 22. 